\title{
A Robust Occlusion Judgment Scheme for Target Tracking Under the Framework of Particle Filter
}

\author{
Kejia Liu, Bin Liu ${ }^{(凶)}$, and Nenghai Yu \\ Key Laboratory of Electromagnetic Space Information Chinese Academy of Sciences, \\ School of Information Science and Technology, \\ University of Science and Technology of China, Hefei, People's Republic of China
} flowice@ustc.edu.cn

\begin{abstract}
In traffic surveillance system, it is still a challenging issue to track an occluded vehicle continuously and accurately, especially under total occlusion situations. Occlusion judgment is critical in occluded target tracking. An occlusion judgment scheme with joint parameters is proposed for target tracking method based on particle filter. A corner matching method is utilized to improve the accuracy of target position and velocity estimation due to structure information, thus obtain a more accurate weight value which can reflect the real target's status. By analyzing the internal relation between the weight value and the particles distribution region based on resample function of particle filter, a new parameter with good performance is proposed to improve the occlusion detection efficiency.
\end{abstract}

Keywords: Target tracking $\cdot$ Particle filter $\cdot$ Robust occlusion judgment $\cdot$ Joint parameters

\section{Introduction}

Object tracking in video has been an active research since more than decades ago. The interest is motivated by numerous applications, such as surveillance, humancomputer interaction, and robots navigation [1]. Although target tracking has been investigated intensively, there are still many challenges, such as occlusions, appearance changes, nonrigid motions, background clutter, etc. Among many challenges in tracking problem, occlusion is one of the most critical issues since it is not straightforward to generalize and model occlusions [2].

A robust target tracking algorithm should be able to detect occlusion status accurately and timely, and keep on tracking the target using the remaining information, which can also predict the target's position reasonably even if the target is totally occluded.

Among the various occluded target tracking strategies, e.g., using multiple cameras [3], training classifier [2], template matching [4], Kalman filter [5], particle filter (PF) [6,7], Mean Shift [8], etc., PF-based tracking algorithms have

(C) Springer International Publishing Switzerland 2015

Y.-J. Zhang (Ed.): ICIG 2015, Part I, LNCS 9217, pp. 423-433, 2015.

DOI: $10.1007 / 978-3-319-21978-3 \_37$ 
been proved to be an effective method for target tracking [9] and is simple, robust and easy to implement [10], especially for vehicle tracking in traffic surveillance scenario with real-time requirement.

In tracking algorithm based on $\mathrm{PF}$, numbers of particles in form of rectangular box are generated to search for target in a certain range according to particles' weight value representing their color feature's similarity to the template. The greater the similarity, the higher the weight. The reference template updated frame by frame might be contaminated by occlusions without proper occlusion detection. As an important part of PF-based tracker, prediction procedure handling total occlusion is often triggered according to an occlusion judgment result. An unreasonable occlusion judgment also deteriorates accuracy of location and velocity estimation, thus degrades tracker's performance.

Researchers have proposed various occlusion judgment method in video tracking base on particle filter. Mao et al. [7] divided target into several patches and judged occlusion by number of matched corners in each patch. However, judgment is adversely affected if corners are unavailable in one of the patches. Mei et al. [11] explored the trivial template coefficients for the occlusion detection. Nevertheless, the above two methods aim to part occlusion but not full occlusion. Yin et al. [12] calculated occlusion probability by patch matching method, and distinguished appearance change and occlusion by Bayesian discriminating function. But it is time cost to acquire right patch with matching method. Gunawan et al. [13] developed a tracking algorithm based on bootstrap PF and color feature by using nonretinotopic concept and they judged occlusion by calculating the sum of color feature similarity. Scharcanski et al. [6] proposed an adaptive particle filter (APF) algorithm, which can handle non-occlusion and total occlusion respectively by defining two different PDFs to generate particles. They also detected occlusion by calculate the similarity between the target and the template based on color feature. Nevertheless, the accuracy of similarity is limited due to randomness of particle's position generated by Bayes' rule and using color feature which discards structure information of target. Moreover, the reliability of a single parameter is susceptible without complementary parameters which implicates occlusion occurrence information.

Corner features, which implicate structure information of object are robust to partial occlusion and illumination, have strong complementarity to color features. ORB [16] which is created based on Rotation-Aware BRIEF and Orientation FAST corner can represent corner rapidly and steadily. Feature from Accelerated Segment Test (FAST) [14] is a corner detector which detects FAST corner by comparing the intensity value between candidate pixel and the circle pixels with certain radius around it. Binary Robust Independent Element Feature (BRIEF) [15] is a bit string description of a image patch constructed from a set of binary intensity tests. In [16], Oriented FAST (OFAST) is presented by orientating FAST corner with intensity centroid of image patch, while Rotated BRIEF (RBEIEF) is proposed by improving BRIEF with a greedy search algorithm. Oriented FAST and Rotated BRIEF (ORB) can then be obtained by constructing RBRIEF on OFAST. 
In this paper, we propose a robust occlusion judgment scheme which adopts corner matching method to improve location accuracy and then obtain a more accurate weight value which represents target status objectively. In addition, a new parameter is created and used in the occlusion judgment scheme to detect occlusion reliably and timely.

Our main contributions are:

(1) An occlusion detection mechanism with joint parameters is designed. The proposed method which is composed of multiple parameters with complementarity is more sensitive to occlusion, has higher discriminability in identifying occlusion status and thus enhance the reliability of occlusion judgment.

(2) A corner matching method is utilized to obtaining precise position as well as parameters such as similarity and weight value, which can reflect target's status more accurately and improve occlusion detection efficiency.

The occlusion detection method of APF is firstly reviewed in Sect. 2, then we describe corner matching method and the proposed occlusion judgment scheme in Sect.3. In Sect.4, the experimental results are discussed and the conclusions are given in Sect. 5 .

\section{Review of Occlusion Judgment in APF}

Scharcanski et al. [6] proposed an adaptive particle filter (APF) algorithm with two tracking mode. Target is tracked by particles obeying the two-dimensional Gaussian distribution and the target's velocity is estimated under non-occlusion. For total occlusion cases, they predicted the object position by particles obeying a joint Normal-Rayleigh bivariate PDF and use the estimated velocity as the parameter of Rayleigh distribution. Let $\boldsymbol{c}_{t}^{i}=\left[x_{t}^{i}, y_{t}^{i}\right]$ denote the center coordinate of the particle's rectangular box in image coordinates and use it to represent the particle. Here $i=1,2, \ldots, N$ means the particle number and $t$ is the frame number.

An occlusion judgment is also designed to switch tracking mode under different occlusion status and described as follows.

Let $\boldsymbol{\Phi}_{P F}$ denote the PF's tracking result, a rectangular box with center coordinate calculated by weighted average of all $\boldsymbol{c}_{t}^{i}$ in the $t$ th frame. $\boldsymbol{\Phi}_{P F}^{u p}$ denotes the tracking result of the previous frame, and the template is represented by $\boldsymbol{\Phi}_{0}$. Let $\phi_{t}, \phi_{t-1}, \phi_{0}$ denote the color histogram of $\boldsymbol{\Phi}_{P F}, \boldsymbol{\Phi}_{P F}^{u p}, \boldsymbol{\Phi}_{0}$ respectively.

Similarity of color histogram is calculated using the Hellinger distance written in terms of the Bhattacharyya coefficient:

$$
\xi_{a, b}=d_{B}\left(\phi_{a}, \phi_{b}\right)=1-B C\left(\phi_{a}, \phi_{b}\right) .
$$

It's correspondingly weight is defined as:

$$
w_{a, b}=\exp \left(-\lambda \times \xi_{a, b}^{2}\right) .
$$

where $\lambda$ is a weight coefficient usually in the range of $[12,20]$. 
Let $w_{t, 0}$ and $w_{t, t-1}$ denote the correspondingly weight of $\xi_{t, 0}$ and $\xi_{t, t-1}$ and $w_{p f m}$ is the minimum of the two weight values:

$$
w_{p f m}=\min \left(w_{t, 0}, w_{t, t-1}\right) .
$$

Thus the occlusion judgment model is designed by comparing $w_{p f m}$ to a predefined threshold. If $w_{p f m}$ is lower than the threshold, a total occlusion judgment is made. Otherwise, it is regarded as non-occlusion status.

There are two demerits in APF. First of all, due to structure information deficiency discarded by color features and limitation of PF's positioning accuracy, the weight value might have weak correlation in representing target status and low occlusion judging quality. Secondly, detecting occlusion is usually unreliable according to single parameter with unsatisfactory separability under different occlusion status, as shown in Fig. 1.

In Fig. 1, we generate the PDF of $w_{p f m}$ based on the video with 30 pixels $\times$ 18 pixels target vehicle and an artificial occlusion of 10 pixels width. We classify values of $w_{p f m}$ into two parts according to occlusion status and acquire their PDF respectively. The classified PDF indicates that it is hard to distinguish them clearly with a single threshold due to the fact that two type of weight values tangle with each other.

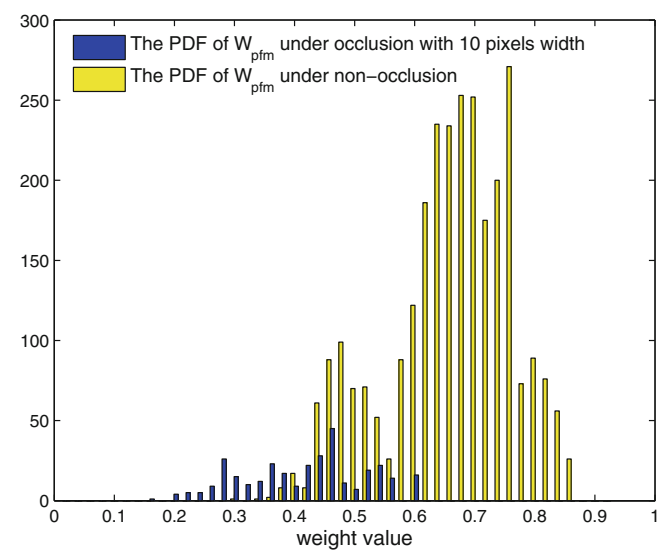

Fig. 1. The classified PDF under two occlusion status

Positioning accuracy and utilization of reliable parameters are helpful in improving occlusion judgment performance. By adopting corner matching method to obtain a more accurate position of target based on $\boldsymbol{\Phi}_{P F}$, a more robust occlusion judgment method with joint parameters is proposed based on particle behavior analysis. 


\section{The Proposed Occlusion Judgment Scheme}

In this section, we present the method of getting preciser target position and weight value, propose a new parameter with good character and the occlusion judgment scheme.

\subsection{Precise Positioning Based on Corner Matching}

As single representation of target, color features used in APF discard structure information, while corner features including structure and position information of object is complementary to color features.

The positioning accuracy grade of PF is limited but always fluctuates in a certain range. Therefore, an ORB-based corner matching method helps to acquire a higher accurate target position through matching corners between $\boldsymbol{\Phi}_{P F}$ and $\boldsymbol{\Phi}_{0}$.

After OFAST corners are respectively detected in $\boldsymbol{\Phi}_{P F}$ and $\boldsymbol{\Phi}_{0}$ by corner detection method in [16], two groups of OFAST corners are matched by calculating the similarity coefficient of their ORB descriptors [16]. OFAST corners are fitted if their ORB similarity exceeds a predefined corner matching threshold. Figure 2 illustrates the corner matching result.

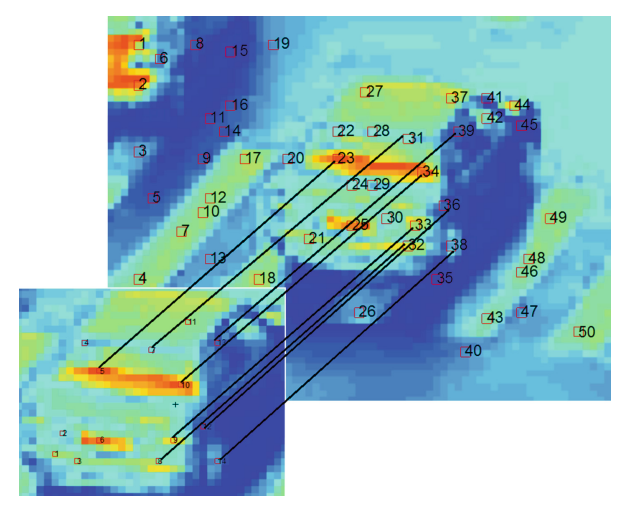

Fig. 2. OFAST corner matching result based on ORB similarity

With the two groups of fitted corners and their coordinates, the target scale can be estimated by comparing the largest external rectangular of two sets of fitted corners. Then, a more accurate position of target can be calculated based on center coordinate of $\boldsymbol{\Phi}_{0}$ and the average coordinate of the fitted-OFAST corners in $\boldsymbol{\Phi}_{0}$ and $\boldsymbol{\Phi}_{P F}$, which is represented by $\boldsymbol{\Phi}_{C M}$. Let $\xi_{c m}$ denotes the similarity of $\boldsymbol{\Phi}_{C M}$ and $\boldsymbol{\Phi}_{0}$ and measured by Bhattacharyya coefficient, the correspondingly weight $w_{c m}$ is given by:

$$
w_{c m}=\exp \left(-\lambda \times \xi_{c m}^{2}\right) .
$$




\subsection{Occlusion Judgment Scheme with Joint Parameters}

During PF's tracking process, particles with higher weight, which means they close to the tracked target, will be reserved and surround the target, and those with lower weight are discarded. Let $P D R$ denote the area covered by these reserved particles in current frame, thus it can be calculated by:

$$
P D R=\frac{1}{N} \sum_{i=1}^{N}\left(\left|x_{t}^{i}-x_{p f}\right|+\left|y_{t}^{i}-y_{p f}\right|\right) .
$$

where $\left[x_{p f}, y_{p f}\right]$ denote the center coordinate of $\boldsymbol{\Phi}_{P F}$. Quantitatively analysis results show that $P D R$ and $w_{c m}$ have strong negative correlation. Thus we can define a new parameter $P P W$ as:

$$
P P W=P D R \times w_{c m}
$$

$P P W$ and $w_{c m}$ are quantitatively analyzed based on video with artificial occlusion in which the target vehicle is a rectangular box with 30 pixels width and 18 pixels height. The classified PDFs of $w_{c m}$ and PPW under occlusion ratio with $33 \%, 66 \%$ and $100 \%$ are illustrated in Fig. 3, respectively.
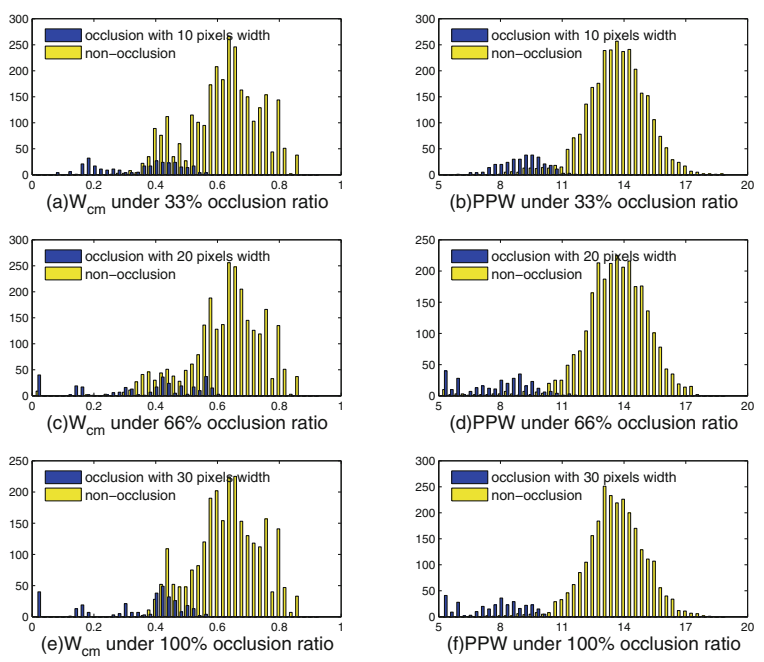

Fig. 3. The classified PDFs of $w_{c m}$ and $P P W$ under different occlusion ratio

As we can see from Fig. 3, the classified PDF of $w_{c m}$ is similar to $w_{p f m}$, where two type of weight values tangle with each other, thus makes distinction works difficult. While PPW obeys normal distribution basically and has an excellent separability in occlusion status under different occlusion ratio due to high accuracy of $w_{c m}$ in representing target and sensitivity of $P D R$ to occlusion. 
Therefore, by joining using three parameters $P P W, w_{c m}$ and $w_{p f m}$, the occlusion judgment scheme with joint parameters is proposed and illustrated in Table 1.

Table 1. Occlusion judgment scheme with joint parameters

\begin{tabular}{|c|c|c|}
\hline Current status & Conditions & Judgment result \\
\hline \multirow[t]{3}{*}{ Under non-occlusion status } & (1) $P P W<T_{p p w}$ & \multirow[t]{3}{*}{$\begin{array}{l}\text { Occlusion is } \\
\text { happening if } \\
\text { one of the } \\
\text { three } \\
\text { conditions is } \\
\text { satisfied }\end{array}$} \\
\hline & (2) $w_{c m}<\min \left(T_{w}, w_{c m}(t-4: t-1)\right)$ & \\
\hline & (3) $w_{p f m}<\min \left(T_{w}, w_{p f m}(t-4: t-1)\right)$ & \\
\hline \multirow[t]{2}{*}{ Under total occlusion status } & (1) $w_{c m}>\max \left(T_{w}, w_{c m}(t-4: t-1)\right)$ & \multirow[t]{2}{*}{$\begin{array}{l}\text { Occlusion is } \\
\text { disappearing if } \\
\text { one of the two } \\
\text { conditions is } \\
\text { satisfied }\end{array}$} \\
\hline & (2) $w_{p f m}>\max \left(T_{w}, w_{p f m}(t-4: t-1)\right)$ & \\
\hline
\end{tabular}

$P P W$ is only used to judge whether an occlusion is happened under nonocclusion status because $P D R$ under occlusion tracking mode has a fixed pattern without target information, and thus make $P P W$ a meaningless variable. $w_{c m}$ can precisely reflects the target status when effective corners exists. $w_{p f m}$ implicating general information about target is still reserved in case that corners are unavailable. The values of $w_{c m}$ and $w_{p f m}$ in the last four frames are also considered since they include variation trend information when occlusion is happening or disappearing. Combination of these parameters can provides more comprehensive information about the target and is beneficial to correct occlusion judgment.

\section{Experimental Result}

All experiments are run on a PC with an Intel Core E7500CPU@2.93GHz. The programming language is MATLAB. We choose two different video sequences as testing sequences, rp9901.avi and rp6401s.avi, as shown in Fig. 4. In the rp9901 sequences, the frame size is $1920 \times 1080$ pixels and a green taxi with 102 pixels width and 101 pixels height is partially occluded by the street lamp and totally occluded by the guidepost. In the rp6401s sequences, the frame size is $640 \times 480$ pixels and a red car with 28 pixels width and 24 pixels height is partially occluded by the light pole and then totally occluded by guidepost. 


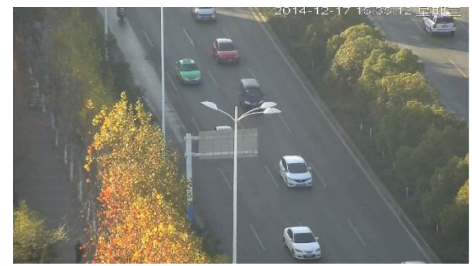

(a)

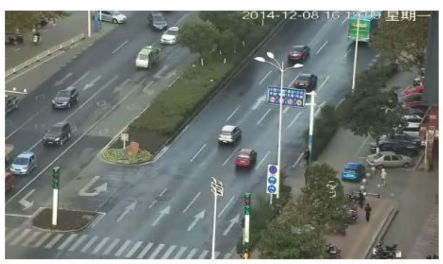

(b)

Fig. 4. Frameshots in experimental videos

We first compare the tracking accuracy and the occlusion judgment parameters between the proposed scheme and the APF. As shown in Fig. 5(a) and (b), the proposed scheme outperforms the APF for both sequences due to the fact that the corner features containing structure information are used and a more appropriate occlusion judgment scheme is adopted. We now get into the details of occlusion judgment parameters as shown in Fig. 5(c) and (d). Occlusion transition is denoted by perpendicular dotted lines. We scale the threshold $T_{p p w}$ with suitable factor to make the parameters comparable with an uniform threshold. Both $w_{c m}$ and $w_{p f m}$ are closed to each other under non-occlusion status while $w_{c m}$ is obviously lower than $w_{p f m}$ under occlusion status, which implicates that $w_{c m}$ is more accurate as a parameter for occlusion detection since totally occluded target is supposed to have lower weight when occlusion and target have different appearance.

We can also see from Fig. 5(c) and (d) that, due to the contribution of $P D R$, $P P W$ could amplify the response of weight clearly and thus predict an occlusion timely and stably. The accuracy of weight and sensitivity of $P D R$ make $P P W$ a steady and reliable parameter in occlusion judgment, which is in accordance with $P P W$ 's separability as shown in Fig. 3 .

Except for corner matching mechanism, the proposed occlusion judgment method can also improve positioning accuracy by detecting occlusion earlier than the single parameter model, thus improve the robustness of tracking algorithm.

Positioning error curves of APF based on two occlusion judgment methods are compared in Fig. 6. Occlusion transition is denoted by perpendicular dotted lines with the same color to their correspondingly curves. The positioning error of APF during the occlusion tracking mode, i.e., between the coupled dotted lines, is obviously lower due to the proposed method.

As shown in Fig. 6(a), the proposed occlusion judgment scheme detects occlusion in the 38th frame which is two frames earlier than APF's, and the correspondingly occlusion ratio is less than $70 \%$. The similar thing happens in Fig. 6(b). On one hand, timely occlusion judgment prevents further deterioration of positioning accuracy, thus helpful in capturing target when occlusion disappearing. On the other hand, it is reasonable to judge total occlusion during $50 \%$ and $70 \%$ occlusion ratio for a moving target as vehicle, since such judgment occasion can avoid hasty erroneous judgment caused by slight occlusion and 


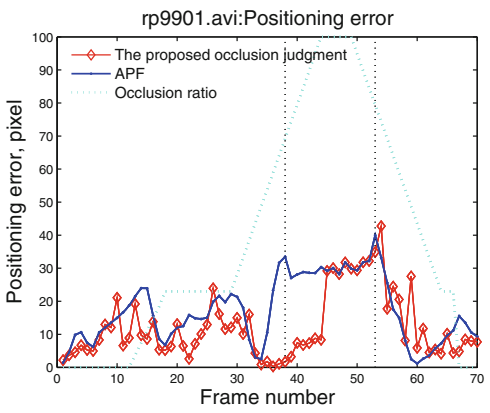

(a)

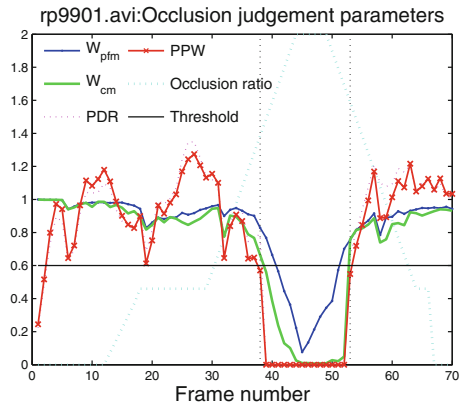

(c)

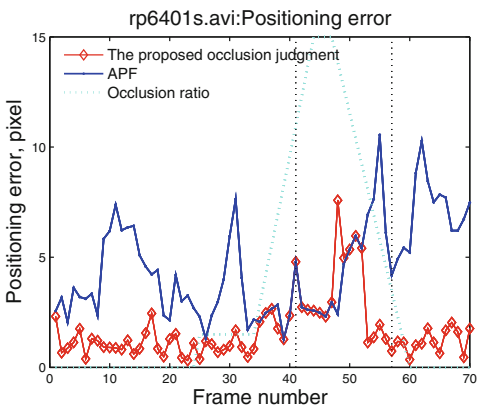

(b)

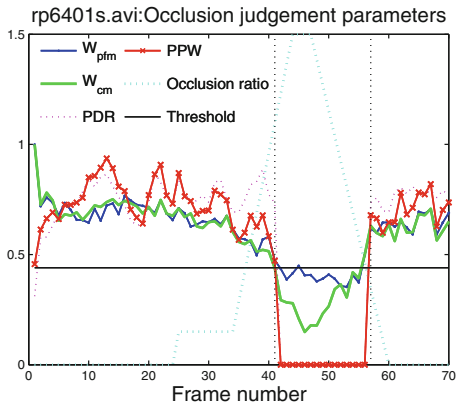

(d)

Fig. 5. Positioning error and the correspondingly occlusion judgment parameters

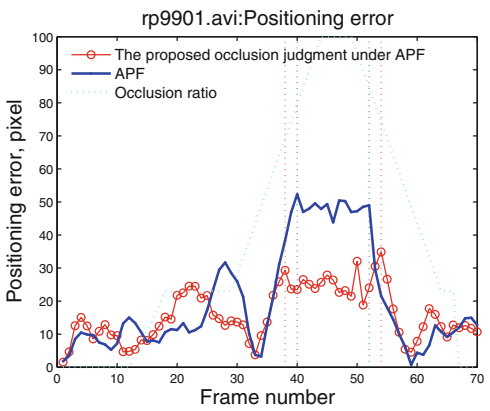

(a)

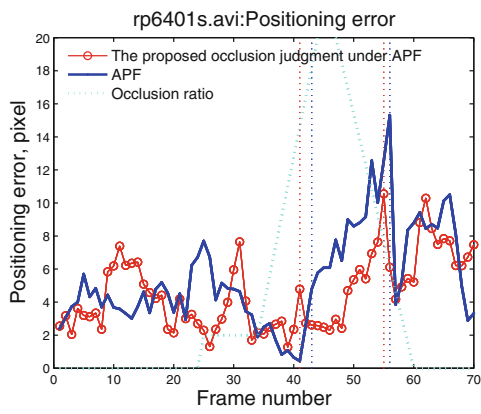

(b)

Fig. 6. The impact of two occlusion judgment models on positioning accuracy of APF 
positioning accuracy deterioration caused by serious occlusion as much as possible. Whereas, $w_{p f m}$ usually make effective detection when target is occluded more than $80 \%$ in both sequences, as a result, positioning error increases due to an tardy judgment.

\section{Conclusions}

A robust occlusion judgment scheme with joint parameters is proposed to detect occlusion timely and steadily. The accuracy of target positioning is improved by ORB-based corner matching due to precise structure information, and a more reliable weight value is obtained which is more accurate in representing target than that of PF's. We also propose a stable parameter with excellent separability in identifying occlusion status. The use of parameters with steadily characters and higher accuracy enhance the reliability of occlusion judgment and improve the tracking algorithm's robustness.

Acknowledgement. This work is supported by the National Natural Science Foundation of China (Grant No. 61371192) and the USTC Grand Master Professor Funds (ZC9850290097).

\section{References}

1. Lee, B.Y., Liew, L.H., Cheah, W.S., Wang, Y.C.: Occlusion handling in videos object tracking: A survey. In: IOP Conference Series, Earth and Environmental Science, Vol. 18, No. 1, pp. 012-020. IOP Publishing (2014)

2. Kwak, S., Nam, W., Han, B., Han, J.H.: Learning occlusion with likelihoods for visual tracking. In: Computer Vision (ICCV), 2011 IEEE International Conference, pp. 1551-1558. IEEE (2011)

3. Fleuret, F., Berclaz, J., Lengagne, R., Fua, P.: Multicamera people tracking with a probabilistic occupancy map. In: Pattern Analysis and Machine Intelligence, IEEE Transactions, 30(2), pp. 267-282 (2008)

4. Jin, L., Wu, T., Liu, F., Zeng, G.: Hierarchical template matching for robust visual tracking with severe occlusions. ZTE Communications 10(4), 54-59 (2012)

5. Jeong, J.M., Yoon, T.S., Park, J.B.: Kalman filter based multiple objects detectiontracking algorithm robust to occlusion. In: SICE Annual Conference (SICE), 2014 Proceedings, pp. 941-946. IEEE (2014)

6. Scharcanski, J., de Oliveira, A.B., Cavalcanti, P.G., Yari, Y.: A particle-filtering approach for vehicular tracking adaptive to occlusions. Vehicular Technology, In: IEEE Transactions 60(2), 381-389 (2011)

7. Mao, W.B., Zheng, J., Li, B.: Patch-based object tracking using corner and color with partial occlusion handling. In: Progress in Informatics and Computing (PIC), 2014 International Conference, pp. 269-274, IEEE (2014)

8. Wen, Z., Cai, Z.: A robust object tracking approach using mean shift. In: Natural Computation, 2007. ICNC 2007. Third International Conference, Vol. 2, pp. 170174. IEEE (2007)

9. Wang, J.J., Li, A.X., Wang, W.Q.: Target Tracking Based on Multi-core Parallel Particle Filtering. In: Computer Science, 8, 065 (2012) 
10. Ma, J., Han, C., Chen, Y.: Efficient visual tracking using particle filter. In: Information Fusion, 2007 10th International Conference, pp. 1-6. IEEE (2007)

11. Mei, X., Ling, H., Wu, Y., Blasch, E.P., Bai, L.: Efficient minimum error bounded particle resampling L1 tracker with occlusion detection. Image Processing, IEEE Transactions 22(7), 2661-2675 (2013)

12. Yin, M.F., Y., Bo, Y.M., Zhao, G.P., Zou, W.J.: Occluded object tracking based on Bayesian Decision theory and particle filtering. In: Control Conference (CCC), 2013 32nd Chinese, pp. 3834-3838. IEEE (2013)

13. Gunawan, A.A., Wasito, I.: Nonretinotopic Particle Filter for Visual Tracking. Journal of Theoretical and Applied Information Technology 63(1), 104-111 (2014)

14. Rosten, E., Drummond, T.: Machine learning for high-speed corner detection. In: Computer VisionCECCV 2006, pp. 430-443. Springer, Berlin Heidelberg (2006)

15. Calonder, M., Lepetit, V., Strecha, C., Fua, P.: Brief: Binary robust independent elementary features. In: Computer VisionCECCV 2010, pp. 778-792. Springer, Berlin Heidelberg. (2010)

16. Rublee, E., Rabaud, V., Konolige, K., Bradski, G.: ORB: an efficient alternative to SIFT or SURF. In: Computer Vision (ICCV), 2011 IEEE International Conference, pp. 2564-2571. IEEE (2011) 